Ophthalmologe 2018 $\cdot 115: 712-713$ https://doi.org/10.1007/s00347-018-0713-1 Online publiziert: 3. Mai 2018

(c) Springer Medizin Verlag GmbH, ein Teil von Springer Nature 2018

CrossMark

\title{
Nicole Eter
}

Universitäts-Augenklinik Münster, Münster, Deutschland

\section{Deep Learning in der Augenheilkunde}

In der Augenheilkunde sind eine elekDer Einsatz künstlicher Intelligenz (KI) in der medizinischen Forschung und im klinischen Alltag hat sehr großes Potenzial, und der Weg hin zur vernetzten, ,intelligenten“ Krankenakte, in der die unterschiedlichen klinischen Befunde, Bildgebung, Diagnosen und Therapien in eine elektronische Krankenakte einfließen, ist in vollem Gange. Durch Integration großer Datenmengen können neue wissenschaftliche Erkenntnisse gewonnen werden. Datenintegration und Datenstandards sind auch wichtige Erfolgsfaktoren für den klinischen Alltag.

》) Die Augenheilkunde könnte bei der digitalen Transformation in der Medizin eine Vorreiterrolle einnehmen

Mithilfe der KI kann die Qualität der Patientenversorgung durch Beschleunigung von Routineaufgaben und durch eine personalisierte Optimierung komplexer klinischer Entscheidungsprozesse deutlich verbessert werden. Umgekehrt können wissenschaftliche KI-Modellbildungen und ihre Implementierung einen sonst nicht zu leistenden Quantensprung auf dem Gebiet der personalisierten Medizin ermöglichen. Durch eine Bündelung der Kompetenzen in aktuellen Informatikverfahren (statistische Klassifikationsverfahren, KI, Machine Learning, neuronale Netze, Deep Learning, Big Data), Schaffung einer vernetzten klinischen Infrastruktur sowie der engen Interaktion zwischen informationstechnologischer Modellierung und klinischer Medizin kann der größtmögliche Nutzen für Patienten und Ärzte aller Fachrichtungen geschaffen werden. tronische Akte und digitale Bildgebung bereits vielerorts umgesetzt. Somit könnte die Augenheilkunde mit der Unterstützung klinischer Abläufe durch digitale Assistenzsysteme eine Vorreiterrolle bei der digitalen Transformation in der Medizin einnehmen.

\section{Machine Learning}

Maschinelles Lernen (ML) ist ein Oberbegriff für die künstliche Generierung von Wissen aus Erfahrung: Ein künstliches System lernt aus Beispielen und kann diese nach Beendigung der Lernphase verallgemeinern. Es werden also nicht einfach Beispiele auswendig gelernt, sondern das System erkennt Muster und Gesetzmäßigkeiten in den Lerndaten. So kann das System danach unbekannte Daten beurteilen (Lerntransfer). Spracherkennung auf Mobiltelefonen oder Tablets wird wesentlich von Machine-LearningAlgorithmen gesteuert. Auch die SpamFilter in Computerprogrammen oder die Gesichtserkennungsprogramme bei Fotos beruhen auf ML. Oft sind wir auch im Kontakt zu lernenden Systemen, ohne es zu wissen - etwa bei der personalisierten Online-Werbung beim Surfen im Internet. In der Medizin findet ML bereits Anwendung bei Sprach- und Texterkennungsprogrammen, automatisierten Diagnoseverfahren sowie Klassifikation von Nukleotidsequenzen. Erste Einsätze gibt es auch in der Augenheilkunde [1].

Das Thema ist eng verwandt mit Knowledge Discovery in Databases und Data-Mining, bei dem es jedoch vorwiegend um das Finden von neuen Mustern und Gesetzmäßigkeiten geht. Viele Algorithmen können für beide Zwecke verwendet werden. Außerdem können Methoden der Knowledge Discovery in Databases genutzt werden, um Lerndaten für "maschinelles Lernen“ $\mathrm{zu}$ produzieren, und Algorithmen aus dem maschinellen Lernen finden wiederum beim sog. Data-Mining Anwendung.

\section{Deep Learning}

Ein erfolgreiches Beispiel künstlicher Intelligenz in der Medizin sind DeepLearning-Algorithmen. Deep Learning (DL) mit neuronalen Netzen ist ein „Megatrend“ in der KI. DL-Systeme verstehen Sprache, ziehen logische Schlüsse und lernen vom Nutzer und von den Daten. Im Unterschied $\mathrm{zu}$ den wissens- und regelbasierten Systemen aus der Anfangszeit der KI-Forschung sind durch die massiv gesteigerte Leistung der Computersysteme jetzt neuronale Netze verfügbar, die eine völlig neue Qualität von Anwendungen in der Medizin ermöglichen. Jüngste Fortschritte in diesem Bereich haben zu wesentlichen Verbesserungen bei einer Reihe von Anwendungen, wie z. B. Bilderkennung, Spracherkennung und Verarbeitung natürlicher Sprache, geführt. Die Potenziale für die Patientenversorgung sind enorm. Verfahren der KI ermöglichen die Entwicklung von digitalen Assistenzsystemen und verleihen ihnen dadurch klinischen Nutzen. Selbstlernenden, kognitiven Computersystemen zur Assistenz bei Diagnostik, Therapieplanung und Patientenmanagement kommt dabei eine entscheidende Rolle $\mathrm{zu}$, insbesondere bei der automatischen Bildanalyse in der medizinischen Bildgebung. Ein Beispiel aus der Dermatologie zeigt: Deep Neural Networks sind in der Melanomdiagnostik teilweise besser als Dermatologen [2]. 
Nach der Radiologie werden in der Augenheilkunde die meisten Bilddaten erzeugt. Daher stellt die Augenheilkunde ein vielversprechendes Feld für den Einsatz von DL-Systemen dar. Bereits 2016 schloss das Moorfields Eye Hospital in London eine Kooperation mit Google Deep Mind zur Analyse retinaler Bildgebung. Eine Vielzahl wissenschaftlicher Gruppen hat sich in den letzten 2 Jahren mit DL in der Ophthalmologie beschäftigt. Experten aus der Medizintechnik und -informatik erwarten viel von dieser Methode. So entstehen weltweit Dutzende Healthcare-Startups, die DL-Methoden einsetzen. In diesem $\mathrm{Zu}$ sammenhang stellt sich jedoch die Frage, wie die Qualität von KI-basierter Bilddiagnostik gesichert werden kann und wie mit Datensammlungen und Auswertungen außerhalb wissenschaftlicher Zentren umgegangen werden soll.

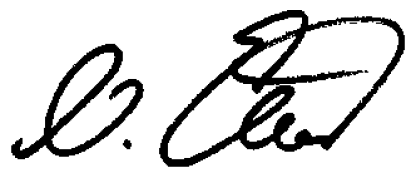

Prof. Dr. Nicole Eter

\section{Korrespondenzadresse}

Prof. Dr. N. Eter
Universitäts-Augenklinik
Münster
Domagkstr. 15, 48149 Müns-
ter, Deutschland
eter@uni-muenster.de

Interessenkonflikt. N. Eter gibt an, dass kein Interessenkonflikt besteht.

\section{Literatur}

1. Rohm M, Tresp V, Müller M, Kern C, Manakov I, Weiss M, Sim DA, Priglinger S, Keane PA, Kortuem K (2018) Predicting visual acuity by using machine learning in patients treated for neovascular agerelated macular degeneration. Ophthalmology. https://doi.org/10.1016/j.ophtha.2017.12.034

2. Esteva A, Kuprel B, Novoa RA, Ko J, Swetter SM, Blau HM, Thrun S (2017) Dermatologist-level classification of skin cancer with deep neural networks. Nature 542(7639):115-118

\section{Immer aktuell - dank „Online-First“-Publikation}

Warum warten, bis die Artikel im Heft abgedruckt sind, wenn Sie sie auch schon vorher lesen können?

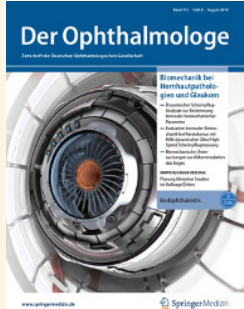

Lesen Sie unter www.springermedizin.de/der-ophthalmologe „Online First Artikel” die aktuellsten Artikel, die gerade online veröffentlicht wurden.

Folgende Übersichten, Originalien, Kasuistiken und „Bild und Fall“Beiträge wurden im vergangenen Monat online publiziert:

- Glaukome im stationären Leistungsgeschehen in Deutschland in 2015

U. Hahn, F. Kretz, J. Koch doi: 10.1007/s00347-018-0757-2

- Ein Patient, dem Hören und Sehen verging A. Rübsam, M. Pahlitzsch, U. Pleyer doi: 10.1007/s00347-018-0758-1

- Morphologische retinale Charakteristika von Patienten mit einer Sehbehinderung infolge eines diabetischen Makulaödems

K. Waser, D. Podkowinski et al. doi: 10.1007/s00347-018-0759-0

- Shaken-Baby-Syndrom bei unilateraler retinaler Hämorrhagie?

I. S. Tarau, X. Wang et al. doi: $10.1007 /$ s00347-018-0764-3

- Stellenwert der Vitrektomie bei Endophthalmitis. Wie eine prompte Vitrektomie den Visus wiederherstellen kann

L.-J. Damm, K. T. Boden, K. Januschowski doi: 10.1007/s00347-018-0768-z

- Vitrektomie mit iOCT-assistierter invertierter ILM-Flap-Technik bei großen Makulaforamina M. Maier, S. Bohnacker et al. doi: 10.1007/s00347-018-0769-y

- Panuveitis infektiologischer Genese C. Lwowski, T. Kohnen doi: 10.1007/s00347-018-0773-2

Übrigens: Die Artikel können mit Angabe des DOI (digital object identifier) und dem Zusatz "epub ahead of print" bereits zitiert werden! 